\title{
Carbon stars in the M 31 dwarf spheroidals: evolutionary implications
}

\author{
D. Harbeck ${ }^{1}$, J.S. Gallagher ${ }^{2}$, E.K. Grebel ${ }^{3}$ and P. Guhathakurta ${ }^{4}$ \\ ${ }^{1}$ UC Berkeley, Space Sciences Laboratory, 7 Gauss Way, Berkeley, CA 94720, USA \\ ${ }^{2}$ University of Wisconsin, Madison, 475 N. Charter Street, Madison, WI 53706, USA \\ ${ }^{3}$ Astronomical Institute of the University of Basel, Venusstr. 7, 4102 Binningen, Switzerland \\ ${ }^{4}$ UCO/Lick Observatory, UC Santa Cruz, 1156 High Street, Santa Cruz, CA 95064, USA
}

\begin{abstract}
We present results of a photometric survey for carbon stars in the M 31 dwarf spheroidal galaxies. From the low frequency of carbon stars in these galaxies compared to their Milky Way cousins we conclude that they consist of predominantly ancient stellar populations. However, the complexity and variety of the metallicity distributions in the M 31 dwarf spheroidals suggest that they still experienced an extended epoch of star formation.
\end{abstract}

Keywords. Local Group, dwarf galaxies, stellar populations

\section{Introduction}

While similar from a distance, the two massive spirals of the Local Group (LG), the Milky Way (MW) and M31, appear different when looked at more closely. For example, the halo of M31 is more metal-rich than that of the MW, suggesting different merger histories of the two systems; M 31 might have experienced a major merger some 7 Gyr ago. In this paper we explore if the differences of the two massive spirals are also reflected in their dwarf galaxy satellite systems. Of particular interest are the dwarf spheroidal (dSph) galaxies, which might be the representation of unused building blocks in hierarchical cold dark matter scenarios of galaxy formation. Their small total and baryonic masses make their star formation history and chemical evolution most susceptible to external regulating mechanisms, such as the onset of reionization or local ram pressure stripping (summarized, e.g., in Grebel et al. 2003). Any local differences between the M 31's and MW's halos might also be unveiled by different episodes of star formation in their satellite galaxies.

The proximity of the MW's dSph galaxies allows a detailed study of their star formation histories based on deep color magnitude diagrams that reach the oldest main sequence turn-off, and, more recently, massive spectroscopic surveys allow one to paint a detailed picture of their chemical evolution. Such an approach is not practical for the M 31 companions, where a reasonable commitment of HST time only allows to obtain photometry to the horizontal branch level. To examine the star formation history of the M 31 companions, in particular their content of intermediate-age stellar populations, we depend on indirect tracers of stellar ages such as luminous AGB stars. For the sparse dSphs galaxies, simple star-counts in the luminosity range of AGB stars are unreliable due to foreground star contamination. However, surveys for carbon (C) stars, AGB stars that have undergone deep mixing, would be less confused by foreground stars due to the distinct spectral features of such stars. C-stars can be readily detected from four-color photometry, by using two broad band filters and two narrow band filters centered on the near infrared $\mathrm{CN}$ and $\mathrm{TiO}$ bands (Cook, Aaronson, \& Norris 1986). Here we report on 

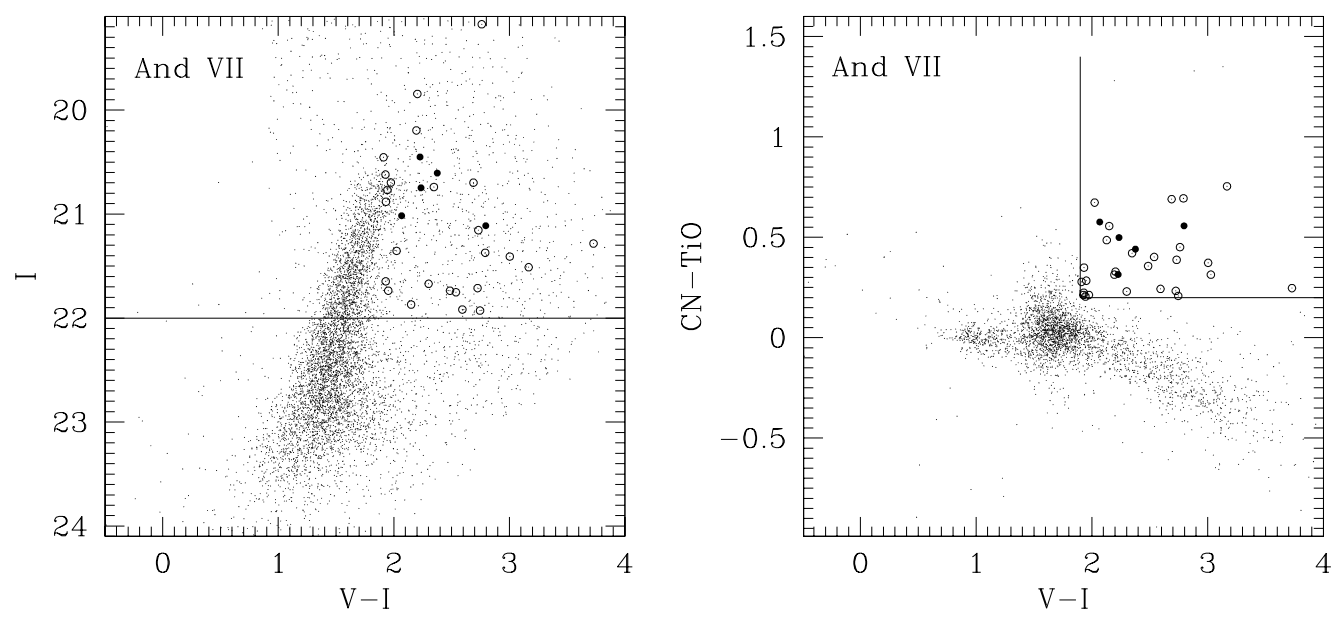

Figure 1. Color-magnitude diagram (left) and two-color diagram (right) of And VII as an example of the survey technique. Open circles show misidentifications due to bright star stray light or cosmic rays. Bona-fide C-stars are drawn with filled circles. Stars above the tip of the red giant branch are considered as C-stars, while less luminous stars are most likely $\mathrm{CH}$ stars, evolved dwarf carbon stars.

our survey for C-stars as a tracer of 1 to 10 Gyr old stellar populations in the M $31 \mathrm{dSphs}$ And III, And V, And VI, And VII, and AND IX with the WIYN 3.5m telescope (details of the survey are described in Harbeck et al. 2004, 2005).

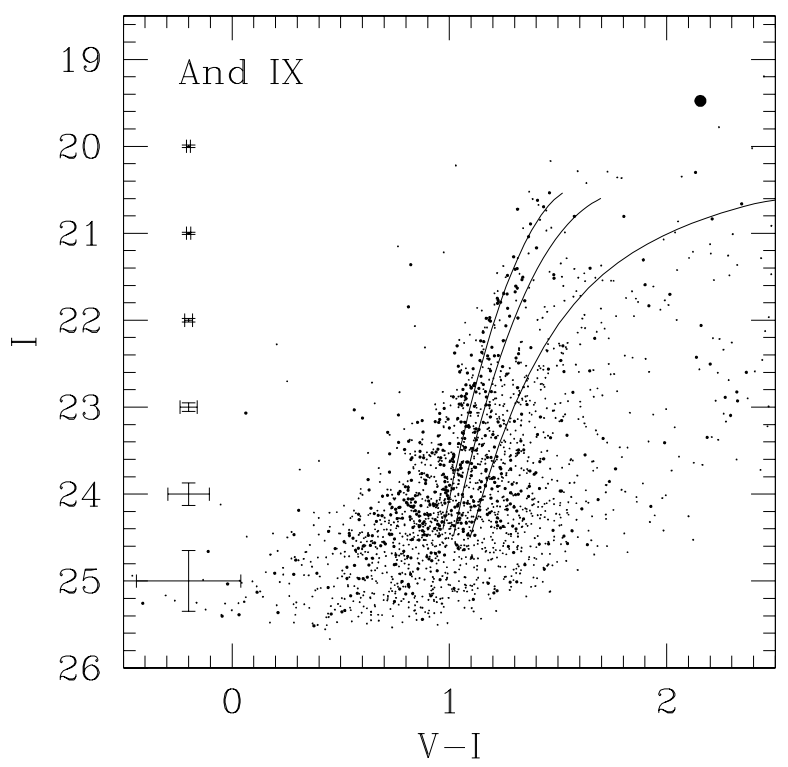

Figure 2. CMD of And IX, based on observations with the WIYN telescope under excellent $\left(0.5^{\prime \prime}\right)$ seeing conditions. Stars within the central arc-minute are plotted with larger dots. We overlay globular cluster fiducials (Da Costa \& Armandroff 1990) with metallicities of -2.26, -1.62, -0.76 dex. And IX seems to be very metal poor, which is expected given its low baryonic mass. The more metal-rich red giant branch of the M 31 halo is visible as well. 


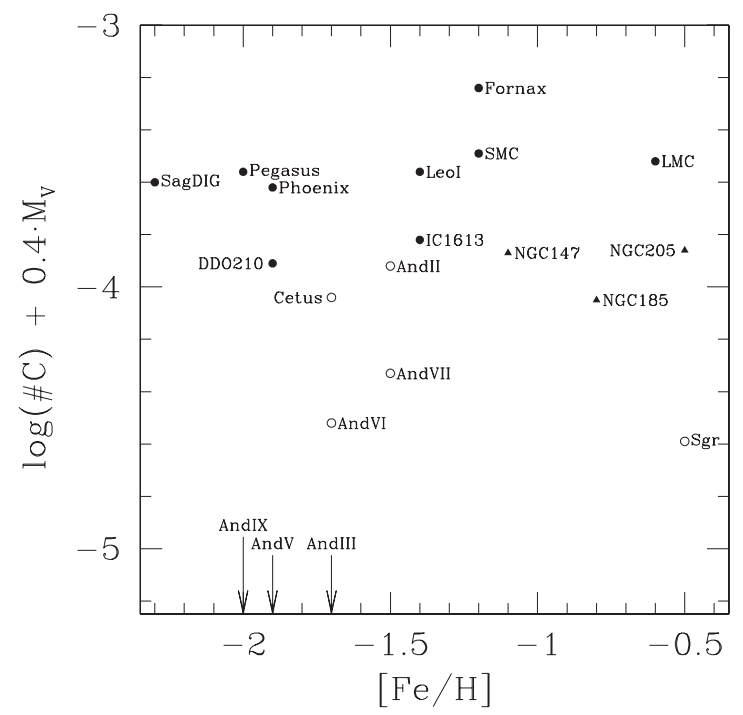

Figure 3. The number of carbon stars - corrected by the host galaxy's luminosity - is compared among Local Group dwarf galaxies. Dwarf galaxies with recent star formation are plotted with filled circles, those with predominantly ancient stellar populations with open circles; triangles refer to dE galaxies. Data for And II taken from Kerschbaum et al. (2004).

\section{WIYN survey for Carbon stars}

The M 31 dSphs galaxies where observed with the WIYN 3.5 meter telescope in Fall 2003 and Fall 2004. Exposure times were $500 \mathrm{~s}$ each in V and I filters and $1500 \mathrm{~s}$ each in the narrow band $\mathrm{TiO}$ and $\mathrm{CN}$ filters. The seeing was always better than $0.8^{\prime \prime}$ and as good as $0.5^{\prime \prime}$. We present a sample color magnitude diagram (CMD) and two color diagram (TCD) of our survey in Fig. 1, where we show the results for And VII. From the color cut in the TCD diagram we select Carbon star candidates that are cross referenced with the original images. False candidates due to bright saturated stars or cosmic rays are rejected. Stars more luminous than the tip of the RGB are regarded as genuine C-stars, while fainter C-stars are probably evolved dwarf carbon ("CH") stars. To summarize our survey results, we find: no C-stars in And III, And V, and And IX; one C-star in And VI; three C-stars in And VII. There is one C-star candidate in the line of sight towards And IX (Fig. 2), however, given the proximity of And IX to M31 this candidate might equally belong to M 31's outer disk.

Fig. 3 compares the number of $\mathrm{C}$ stars normalized by the luminosity (i.e., baryonic mass) of their host galaxy of Local Group dwarf galaxies. We concentrate on those galaxies where surveys are believed to be complete (see Harbeck et al. 2004 for a description of the sample). Two conclusions can be drawn from this graph: (i) compared to the MW dSph galaxies, the And companions appear under-abundant in C-stars, i.e., there are no Fornax-type dSphs among the Andromeda dSphs. It appears that the M $31 \mathrm{dSphs}$ contain predominantly ancient (i.e., older than $10 \mathrm{Gyr}$ ) stellar populations. The M $31 \mathrm{dSph}$ show no imprint of M31's major merging event. (ii) In particular with And II, there seems to be sufficient overlap in the C-star frequency in the M 31 and MW dSphs to conclude that their nature is not substantially different. 


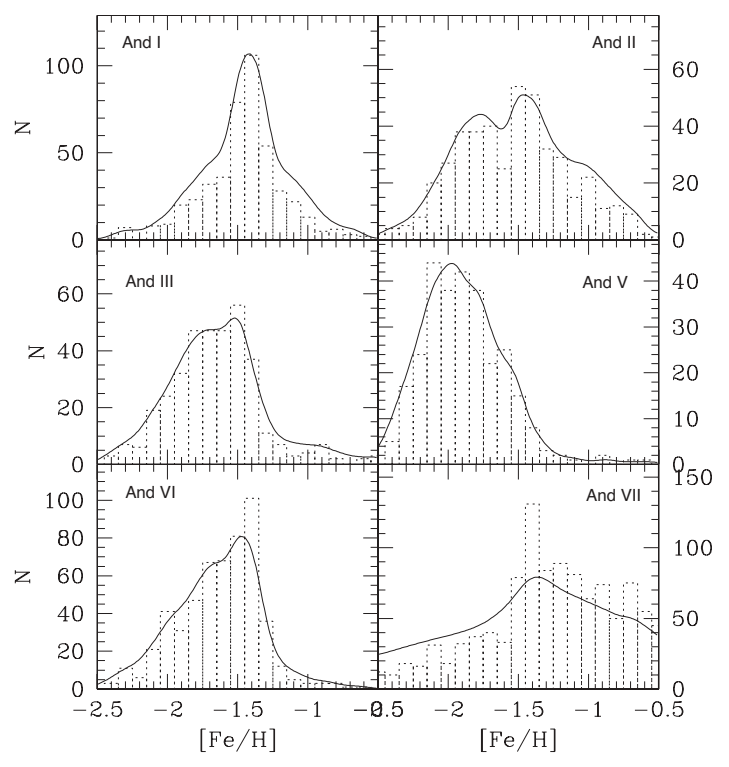

Figure 4. Metallicity distribution of M 31 dSphs galaxies based on RGB star colors (derived from HST photometry, see Harbeck et al. 2001 for details). The distribution of And VII is dominated by photometric errors. The variety and broadness of the metallicity distributions suggests very different, partly complex star formation histories of these galaxies.

\section{Ancient, but not trivial}

The under-abundance of C-stars in the M 31 dSphs suggests a fast decline of star formation activities in these galaxies compared to their Milky Way cousins. However, this does not imply simple enrichment histories. In Fig. 4 we plot the stellar metallicity distributions of M 31 dSphs derived from HST photometry. These metallicity distributions are calculated by comparing colors of red giant branch stars to globular cluster fiducials (Da Costa \& Armandroff 1990). We are assuming predominantly old stellar populations in the dSphs (which we have seen to be justified for most M 31 dSphs - maybe with the exception of And II). The variety of different metallicity distributions among the M 31 $\mathrm{dSphs}$ is apparent, emphasizing that each galaxy is an individual case. To achieve a broad metallicity distribution as it is seen most of the M $31 \mathrm{dSphs}$ an extended (e.g., at least 4 Gyr) period of star formation is required (Ikuta \& Arimoto 2002).

\section{Conclusions}

C-stars offer a possibility to derive a rough picture of a galaxy's intermediate-age star formation history where deep photometry is not available; at least a classification into galaxies dominated by old or intermediate stellar populations is possible. The apparent under-abundance of C-stars in the M 31 dSphs suggests a faster decline of star formation activity compared to the MW dSphs. The M 31 dSphs' stellar populations are both older and more metal-poor than the M31 halo; we thus conclude that they are indeed an primordial legacy and are not tidal dwarfs produced during a later major merger event. And II and And VII, with a moderate content of C-stars and broad metallicity might most closely resemble Galactic dSphs with extended epochs of star formation. 


\section{Acknowledgements}

DH gratefully acknowledges support by an IAU travel grant.

\section{References}

Cook, K.H., Aaronson, M. \& Norris, J. 1986, ApJ 305, 634

Da Costa, G.S. \& Armandroff, T.E. 1990, AJ 100, 162

Grebel, E.K., Gallagher, J.S. \& Harbeck, D. 2003, $A J$ 125, 1926

Harbeck, D. et al. 2001, AJ 122, 3092

Harbeck, D., Gallagher, J.S. \& Grebel, E.K. 2004, AJ 127, 2711

Harbeck, D., Gallagher, J.S., Grebel, E.K., Koch, A. \& Zucker, D.B. 2005, ApJ 623, 159

Ikuta, C. \& Arimoto, N. 2002, A\&A 391, 55

Kerschbaum, F., Nowotny, W., Olofsson, H. \& Schwarz, H.E. 2004, A\& A 427, 613

\section{Discussion}

FERGUSON: If you assume all the stars were the same age, how young could you push the age without violating your carbon star constraint?

HARBECK: C-stars ( and the lack of them) constrain atellar populations with ages from 10 Gyr to 1 Gyr. Stellar populations younger than 1 Gyr can be excluded from existing CMDs. What happens within the 1-10 Gyr range cannot be constrained by just counting C-stars.

Conselice: Could the higher number of Carbon stars in the Milky Way be the result of contamination, as the field of view needed to observe the Milky Way system is larger?

HARBECK: At the luminosity of MW dSph Carbon stars, potential contaminators could only be dwarf Carbon (dC) stars. These, however, are very rare and are unlikely to be a significant contaminator. As $\mathrm{dC}$ stars are faint, they would be nearby and could be identified by their proper motions.

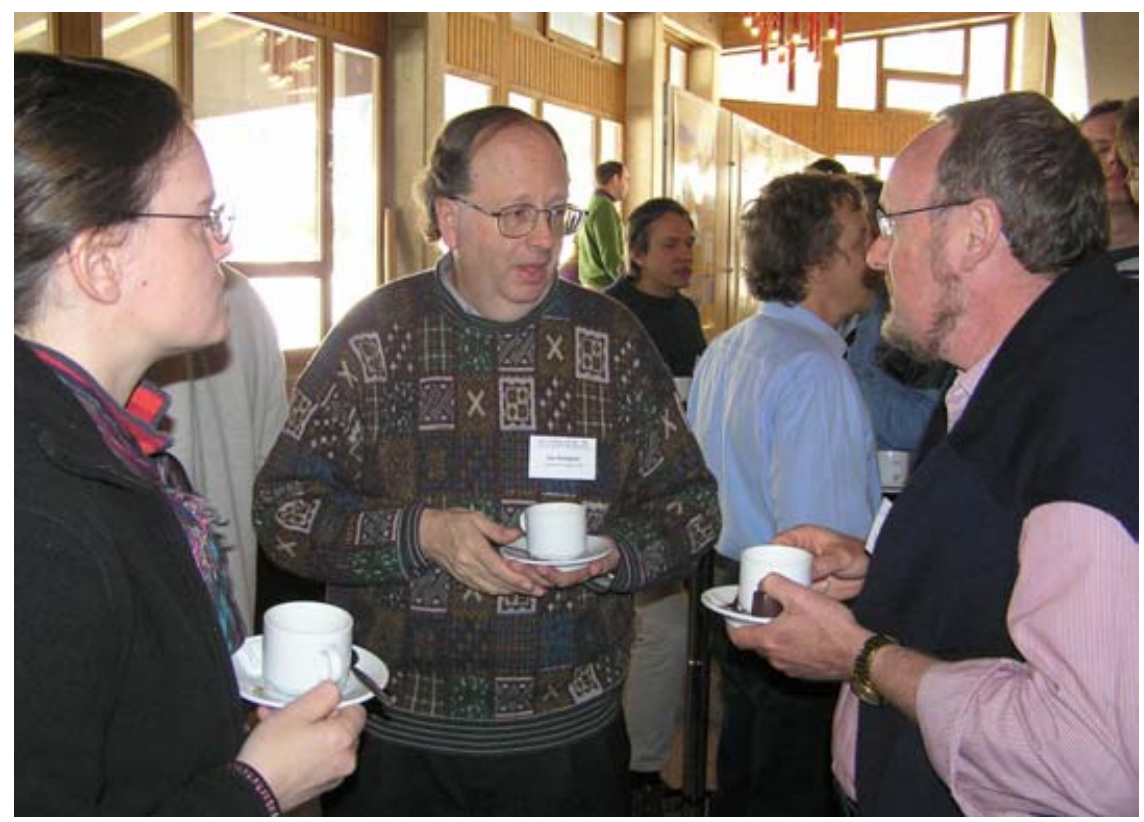

